\title{
Empirical Mode Decomposition (EMD) for Platform Motion Compensation in Remote Life Sensing Radar
}

This paper was downloaded from TechRxiv (https://www.techrxiv.org).

LICENSE

CC BY 4.0

SUBMISSION DATE / POSTED DATE

$25-07-2021 / 30-07-2021$

\section{CITATION}

Islam, Shekh Md Mahmudul (2021): Empirical Mode Decomposition (EMD) for Platform Motion Compensation in Remote Life Sensing Radar. TechRxiv. Preprint. https://doi.org/10.36227/techrxiv.15049734.v1

$\mathrm{DOI}$

10.36227/techrxiv.15049734.v1 


\section{Empirical Mode Decomposition (EMD) for Platform Motion Compensation in Remote Life Sensing Radar}

\author{
Shekh M. M. Islam \\ dept. of Electrical and Electronic \\ Engineering \\ University of Dhaka \\ Dhaka, Bangladesh \\ shekh@hawaii.edu
}

\author{
Lupua Oba \\ dept. of Electrical and Computer \\ Engineering \\ University of Hawaii at Manoa \\ Honolulu, HI, USA \\ loba@hawaii.edu
}

\author{
Victor M. Lubecke \\ line 2: dept. of Electrical and Computer \\ Engineering \\ University of Hawaii at Manoa \\ Honolulu, HI, USA \\ lubecke@hawaii.edu
}

\begin{abstract}
Radar sensing of respiratory motion from unmanned aerial vehicles (UAVs) offers great promise for remote life sensing especially in post-disaster search and rescue applications. One major challenge for this technology is the management of motion artifacts from the moving UAV platform. Prior research has focused on using an adaptive filtering approach which requires installing a secondary radar module for capturing platform motion as a noise reference. This paper investigates the potential of the empirical mode decomposition (EMD) technique for the compensation of platform motion artifacts using only primary radar measurements. Experimental results demonstrated that the proposed EMD approach can extract the fundamental frequency of the breathing motion from the combined breathing and platform motion using only one radar, with an accuracy above $87 \%$.
\end{abstract}

Keywords—empirical mode decomposition, UAV, radar, motion artifacts.

\section{INTRODUCTION}

Unmanned aerial vehicles (UAV), popularly known as drones, are emerging as a valuable tool for reconnaissance, search-and-rescue applications, emergency medical services, and food delivery [1]. At present, rapid triage assessment has been performed by UAVs relying primarily on optical imagery [2-3]. A UAV-borne radar motion sensor can potentially increase the reconnaissance capabilities as it can detect human breathing and heart rate even through debris, poor lighting, fog, smoke, and clothing [4]. Non-Contact vital signs monitoring using stationary microwave Doppler radar has shown efficacy and potential in various healthcare applications [5] and is gaining attention for security surveillance applications including recognition of individuals from their breathing diversity [6]. Additionally, radar has been proposed as a powerful tool for finding trapped or injured people in post-disaster rescue scenarios by integrating it with the UAV platform [7]. One of the key challenges is that the mobile UAV platform motion creates extraneous motion that adds an interfering phase component to the radar baseband signal which makes it difficult to extract vital signs [7-8].

Prior UAV-borne vital sign sensing research has focused on using the direction of arrival (DOA) which requires motion stabilization that is challenging in a realistic setting [9]. In a prior study, an adaptive filter technique for UAV-borne radar motion compensation was demonstrated with preliminary results obtained for a testbed using a robotic mover to simulate drone and breathing motion [7-8]. One significant limitation of the adaptive filter technique is the requirement of an additional secondary radar for providing noise reference signals. Moreover, for search-and-rescue applications, mounting secondary radar would be challenging and there may be no ceiling to aim the reference radar (Fig. 1). Therefore, a reliable and robust platform motion compensation technique is required to extract vital signs without the use of a secondary radar module.

This paper investigates the efficacy of using the empirical mode decomposition (EMD) technique to isolate a breathing motion pattern from platform motion using only a single radar, as an alternative to previously demonstrated dual radar ANC techniques [7-8]. The use of robotic phantom mover [10] and EMD technique has been well established [11]. The prior reported results of the EMD technique is based on the foreknowledge of the signal [11] and the proposed technique does not rely on the separate measurement of the signal as it can extract the best intrinsic mode function based on a signalto-noise ratio (SNR) when the subject's breathing motion can be assumed to be larger than the radar platform motion.

\section{THEORETICAL BACKGROUND}

The UAV platform motion creates artifacts in the radarcaptured respiration pattern. The combined motion due to the platform and respiration motion detected by the radar sensor is represented as [7-8]:

$$
S_{\text {composite }}(t)=\cos \left(\frac{4 \pi A}{\lambda} \sin \left(\omega_{1} t\right)+\frac{4 \pi B}{\lambda} \sin \left(\omega_{2} t\right)\right),
$$

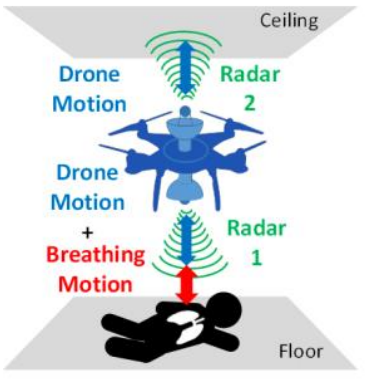

(a)

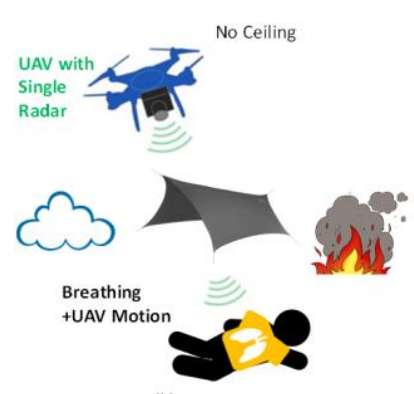

Fig. 1 (a) Concept for an indoor search and rescue UAV where secondary radar is mounted on the top facing the ceiling to provide a noise reference for adaptive filtering [7-8]. (b) Outdoor search and rescue scenario where there is no ceiling thus making a noise measurement with a second radar more difficult, and where EMD can potentially be used to separate breathing motion from interfering UAV motion. 
Where, $\omega_{1}$ is the angular frequency for the chest surface movement and $\omega_{2}$ is the undesired platform motion, which needs to be removed to extract vital signs information. The indoor drone radar concept is shown in Fig. 1(a) and the outdoor drone radar concept is illustrated in Fig. 1 (b), where a noise measurement cannot be easily made without a ceiling.EMD is used to decompose the demodulated radar signal into two different components. One component is the physiological motion and the other component is the platform motion-related interference [11]. EMD, in general, decomposes the signal as intrinsic mode functions (IMFs) and residuals represented as:

$$
x[n]=\sum_{k=1}^{n} d_{k}[n]+r[n] ;
$$

where $d_{k}[n]$ denotes an IMF First. The best IMF is selected based on the comparison of the SNR. SNR is calculated with the Matlab platform utilizing the SNR function based on the summed squared power of the periodogram of the signal with the modified periodogram of the same length signal. When the extracted IMF contains more breathing-related information the SNR is higher.

\section{MEASUREMENT SETUP}

In prior work, the feasibility of the adaptive filter technique was tested for an indoor drone radar scenario by building a testbed of robot mover measurements as shown in Fig. 2 (a) [7-8] Two 24-GHz KLC-1LP monopulse radar modules, each with two channels (I, Q) and connected to Low noise amplifier (LNA) SR560, was used. LNA's are accoupled with a gain of 500 and a cut-off frequency of $3 \mathrm{~Hz}$. Finally, the LNA's output was connected to a NI DAQ which is connected to the computer through an interface. A customized MATLAB recording interface was used to record the signals. Here in Fig. 2(b) it is shown that the two radar

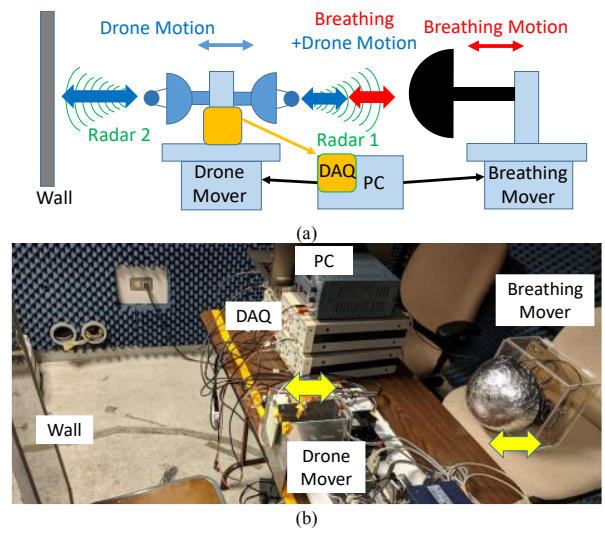

Fig. 2 (a) Concept of simulated drone motion and breather motion, with two radars in a testbed [7-8]. (b) Experimental measurement setup where primary radar is pointed towards a breathing mover and the secondary radar is pointed towards a reference surface for collecting reference noise. From [7-8]

modules are mounted on a linear actuator with one pointing towards a simulated breather [10], and the other to a reference surface, simulating indoor overhead cover.

\section{RESULTS}

For implementing the proposed EMD approach, radar waveforms which are represented in the time domain shown in Fig. 3 were captured. There were three distinct segments in the captured data. The first 3 minutes represent the breathinglike motion of the phantom mover. During this time, the phantom mover was moving without any interference at a

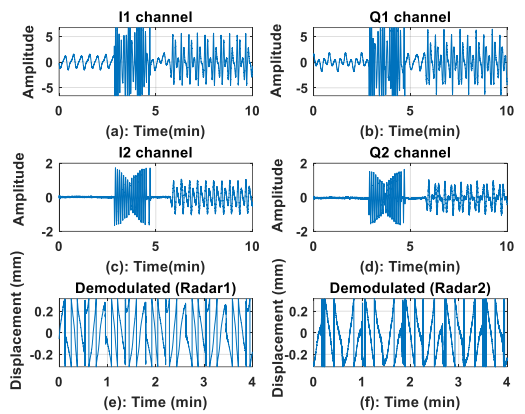

Fig. 3 Radar captured motion of four different channels (a) primary radar I1, (b) primary radar I2, (c) secondary radar Q1, and (d) secondary radar Q2. Demodulated combined mixture of drone and radar movement (e) primary radar (f) secondary radar.

frequency of $0.2 \mathrm{~Hz}$ and a displacement of $0.5 \mathrm{~cm}$. The sampling rate was $500 \mathrm{~Hz}$ with a range of -10 to $+10 \mathrm{~V}$. This clean reference breathing motion was utilized for measuring the accuracy of the EMD approach. Then drone movement was initialized from 3 to 5 minutes shown in Fig. 3. The segmented portion of the combined mixture of drone movement and breather movement of the I and Q channel signals was demodulated using arc-tangent demodulation to extract the maximum displacement information. Fig. 4 illustrates the 9 IMFs and residual signals from the combined signal. As can be seen from Fig. 4, IMF1 and IMF2 show the interference signal or mostly platform motion. To select the best IMF initially the IMFs were visually inspected and SNR assessed. Table-I represents the SNR of all IMFs used for the

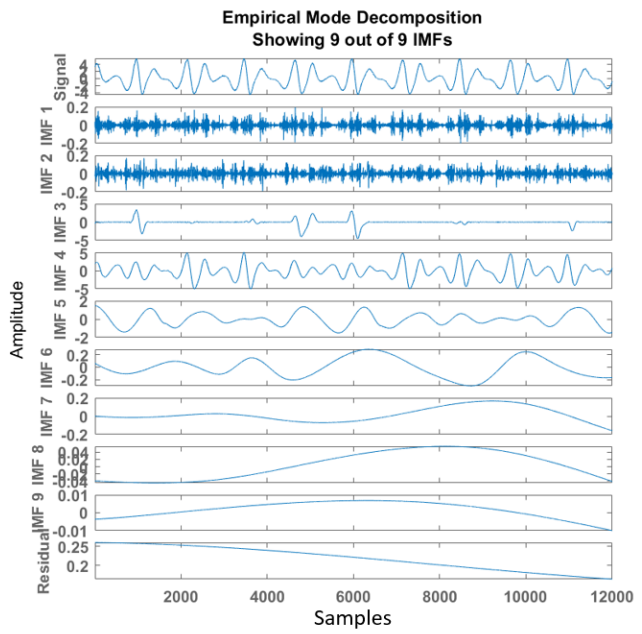

Fig. 4 Intrinsic mode function (IMF) as a result of computing the EMD of the combined signal from primary radar. IMF1 and IMF2 have high frequency content represents the interferences. IMF 3-6 illustrates compatible with the breathing motion.

TABLE I

SUMMARY OF ACCURACY FOR BREATHING PATTERNS

\begin{tabular}{|c|c|}
\hline IMFs & SNR (dB) \\
\hline IMF1 & $-17.64 \mathrm{~dB}$ \\
\hline IMF2 & $-13.48 \mathrm{~dB}$ \\
\hline IMF3 & $-2.89 \mathrm{~dB}$ \\
\hline IMF4 & $0.38 \mathrm{~dB}$ \\
\hline IMF5 & $19.45 \mathrm{~dB}$ \\
\hline IMF6 & $64.52 \mathrm{~dB}$ \\
\hline IMF7 & $14.15 \mathrm{~dB}$ \\
\hline IMF8 & $4.81 \mathrm{~dB}$ \\
\hline IMF9 & $23.45 \mathrm{~dB}$ \\
\hline
\end{tabular}


selection of the best candidate which carries the breather frequency-related information. From Table-I, it is clear that IMF6 has the best SNR. A fast Fourier transform (FFT) was then performed for all extracted IMFs to find their highest frequency component. Fig. 5 shows the FFT of four different IMFs. From Fig. 5 the FFT of IMF1, IMF3, IMF6, and IMF 7 shows the highest frequency content as $0.67 \mathrm{~Hz}, 2.83 \mathrm{~Hz}, 0.17$ $\mathrm{Hz}$, and $1.7 \mathrm{~Hz}$ respectively. It is also clear that the FFT of IMF1 shows multiple peaks in the frequency domain plot which also validates that the particular IMF contains platform motion-related information. On the other hand, the FFT plot of IMF6 shows only a single peak in the frequency domain and it closely matches the breather phantom mover fundamental frequency components. For testing the reproducibility and repeatability of the proposed approach, the experiments were repeated where breathing frequency was simulated as a sinusoidal pattern with a frequency of $0.2 \mathrm{~Hz}$ to a maximum of $0.5 \mathrm{~Hz}$. Three different breathing depths were selected such as $3.75,5$, and $10 \mathrm{~mm}$. Similarly, the motion of the drone was simulated as a sinusoid with a frequency of 1.8 $\mathrm{Hz}$ and an amplitude of $10 \mathrm{~mm}$ peak-to-peak. After finding the best IMF based on the SNR, we performed the FFT of the best IMF to extract the highest frequency content. The highest frequency content was compared with the reference breather movement FFT and the accuracy was calculated based on below equations:

$$
\text { Accuracy }(\%)=\frac{\mid \text { actual frequency }- \text { reconstructed frequency } \mid}{\text { actual frequency }}
$$

Fig. 6 illustrates the frequency domain plot of the best IMF and the breather motion. It is shown that the best IMF reconstructed frequency is $0.1742 \mathrm{~Hz}$ whereas the phantom breather frequency is around $0.2 \mathrm{~Hz}$. The accuracy of the proposed system for varying frequencies remained above $87 \%$. Testing on more realistic UAVs and breathing motion is expected to produce similar results, yet remains beyond the scope of the current experiment.
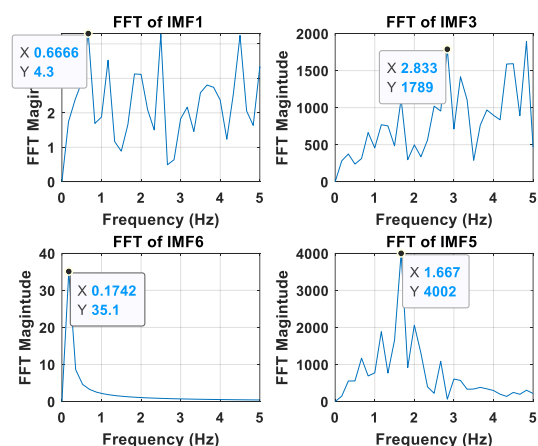

Fig. 5 FFT of the intrinsic mode function (IMF) after computing EMD. Here it represents the FFT of four IMFs (IMF1, IMF3, IMF6, and IMF5).

\section{CONCLUSION}

In this paper, the feasibility of using the EMD method for isolating the breather frequency from the combined drone and breathing movement using a single radar was evaluated. From the experimental results, it was demonstrated that by incorporating the EMD method in a moving platform radar, breathing frequency can be extracted from the combined motion.

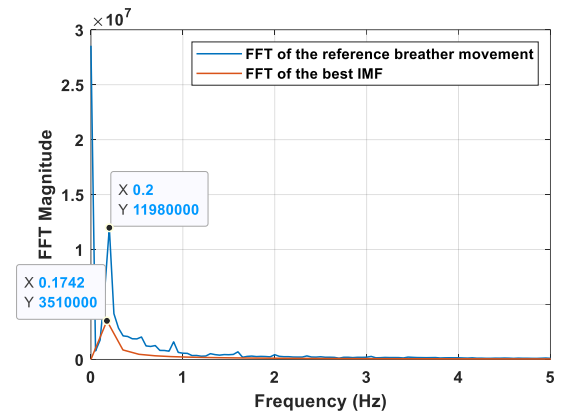

Fig. 6 Compariosn of reference breather motion FFT and FFT of the best IMF shown in frequency domain. The peak of theFFT of the best IMF is around $.1742 \mathrm{~Hz}$ whereas the breather reference frequency is around $.2 \mathrm{~Hz}$.

\section{REFERENCES}

[1] N. Zhao et al., "UAV-Assisted Emergency Networks in Disasters," IEEE Wireless Commun., vol. 26, no. 1, pp. 45-51, Feb. 2019, doi: 10.1109/MWC.2018.1800160.

[2] H. Shakhatreh et al., "Unmanned Aerial Vehicles: A Survey on Civil Applications and Key Research Challenges," IEEE Access, vol. 7, pp. 48572-48634, 2019, doi: 10.1109/ACCESS.2019.2909530.

[3] Y. Rong, R. M. Gutierrez, K. V. Mishra, and D. W. Bliss, "NonContact Vital Signs Detection with UAV-Borne Radars," arXiv:2011.13982 [eess], Nov. 2020, Accessed: Mar. 11, 2021. [Online]. Available: http://arxiv.org/abs/2011.13982.

[4] K.M. Chen, Y. Huang, J. Zhang, A. Norman, “ A Microwave lifedetection systems for searching human subjects under earthquake rubble or behind barrier", IEEE Trans. Biomed. Eng. 2000; 47:105114. doi: $10.1109 / 10.817625$.

[5] C. Li, V. M. Lubecke, O. Boric-Lubecke, and J. Lin, "A Review on Recent Advances in Doppler Radar Sensor for Non-contact Healthcare Monitoring", IEEE Trans. Microwave Theory Techn., vol. 61, non. 5, pp. 2046-2060, May 2013, doi: 10.1109/TMTT.2013.2256924.

[6] S. M. M. Islam, O. Borić-Lubecke, Y. Zheng, and V. M. Lubecke, "Radar-Based Non-Contact Continuous Identity Authentication," Remote Sensing, vol. 12, no. 14, p. 2279, Jul. 2020, doi: $10.3390 /$ rs 12142279 .

[7] S. M. M. Islam, L. C. Lubecke, C. Grado, and V. M. Lubecke, "An Adaptive Filter Technique for Platform Motion Compensation in Unmanned Aerial Vehicle-Based Remote Life Sensing Radar," in 2020 50th European Microwave Conference (EuMC), Utrecht, Netherlands, Jan. 2021, pp. 937-940, doi: 10.23919/EuMC48046.2021.9338011.

[8] S. M. M. Islam, C. Grado, V. Lubecke, and L. C. Lubecke, "UAV Radar Sensing of Respiratory Variations for COVID-Type Disorders," in 2020 IEEE Asia-Pacific Microwave Conference (APMC), Hong Kong, Hong Kong, Dec. 2020, pp. 737-739, doi: 10.1109/APMC47863.2020.9331613.

[9] R. H. Nakata, B. Haruna, T. Yamaguchi, V. M. Lubecke, S. Takayama, and K. Takaba, "Motion Compensation for an Unmanned Aerial Vehicle Remote Radar Life Sensor," IEEE J. Emerg. Sel. Topics Circuits Syst., vol. 8, no. 2, pp. 329-337, Jun. 2018, doi: 10.1109/JETCAS.2018.2821624.

[10] S. M. M. Islam, B. Tomota, A. Sylvester, and V. M. Lubecke, "A Programmable Robotic Phantom to Simulate the Dynamic Respiratory Motions of Humans for Continuous Identity Authentication," in 2019 IEEE Asia-Pacific Microwave Conference (APMC), Singapore, Singapore, Dec. 2019, pp. 1408-1410, doi: 10.1109/APMC46564.2019.9038224.

[11] I. Mostafanezhad, O. Boric-Lubecke, V. Lubecke, and D. P. Mandic, "Application of empirical mode decomposition in removing fidgeting interference in doppler radar life signs monitoring devices," in 2009 Annual International Conference of the IEEE Engineering in Medicine and Biology Society, Minneapolis, MN, Sep. 2009, pp. 340-343, doi: 10.1109/IEMBS.2009.5333206. 